EPJ Web of Conferences 41, 09011 (2013)

DOI: $10.1051 /$ epjconf/20134109011

C) Owned by the authors, published by EDP Sciences, 2013

\title{
Recovery of ultra-broadband terahertz pulses from sum- frequency spectrograms using a generalized deconvolution method
}

\author{
Mark D. Thomson ${ }^{1, a}$, Volker Blank ${ }^{1}$, and Hartmut G. Roskos ${ }^{1}$ \\ Physikalisches Institut, Johann Wolfgang Goethe-Universität, Max-von-Laue-Str. 1, 60438 Frankfurt, \\ Germany
}

\begin{abstract}
A method to recover the intensity and phase of ultra-broadband $\mathrm{THz}$ pulses exceeding $100 \mathrm{THz}$ bandwidth is presented, using a generalized deconvolution algorithm which incorporates an arbitrary phase-matching and non-linear response.
\end{abstract}

\section{Introduction}

The generation of ultra-broadband terahertz $(\mathrm{THz})$ pulses, extending well into the mid-infrared range $(>100 \mathrm{THz})$, allows one to access new spectroscopic regimes in the study of non-linear and ultrafast phenomena. One method that can readily yield such $\mathrm{THz}$ pulses employs a laser-induced gas plasma with two-color $(\omega-2 \omega)$ optical pump pulses [1]. A key challenge for experiments is the ability to recover the full temporal intensity and phase profile of the $\mathrm{THz}$ pulses, i.e. using coherent detection. While electro-optic time-domain sampling has been successfully refined for bandwidths reaching 100 $\mathrm{THz}$ [2] (and provides the absolute phase), beyond this the requirements for the optical detection pulse duration become severe. Here we present a spectrogram detection method, where a sum-frequency signal between the $\mathrm{THz}$ and an optical detection pulse is measured vs. delay and signal frequency, which relaxes the requirement on the optical pulse duration (albeit at the expense of the absolute phase). In order to enable recovery of the underlying ultra-broadband $\mathrm{THz}$ pulse, we have developed a generalized deconvolution algorithm, which allows for the incorporation of the complex bi-spectral phase-matching and non-linear response. The potential of the algorithm is demonstrated by recovery of a known $\mathrm{THz}$ pulse from a numerically generated spectrogram.

\section{Sum-frequency THz-optical spectrograms}

These methods are essentially cross-correlation frequency-resolved optical gating (XFROG), where algorithms for the recovery of the underlying unknown pulse are well established for the optical range. For ultra-broadband pulses, the effect of phase-matching and the dispersion of the non-linearity cannot be neglected. For all-optical XFROG, two methods have been introduced to account for this, either (i) by applying an approximate correction vs. signal wavelength to the measured FROG trace [3], or (ii) by dithering the angle of the birefringent non-linear crystal in order to produce an average signal where phase-matching is sufficiently achieved across the bandwidth of interest [4]. Unfortunately, such methods are not readily applicable for extreme bandwidths covering the THz-mid-IR range, especially in conjunction with an ultra-short optical detection pulse (i.e. $<20 \mathrm{fs}$ ). In this case, the spectrogram signal at each frequency $(\Omega)$ comprises contributions from a broad range of THz-optical frequency pairs, with a significant variation of the phase-matching factor and non-linear susceptibility (especially

\footnotetext{
a e-mail: thomson@physik.uni-frankfurt.de
}

This is an Open Access article distributed under the terms of the Creative Commons Attribution License 2.0, which permits unrestricted use, distribution, and reproduction in any medium, provided the original work is properly cited. 
(a)

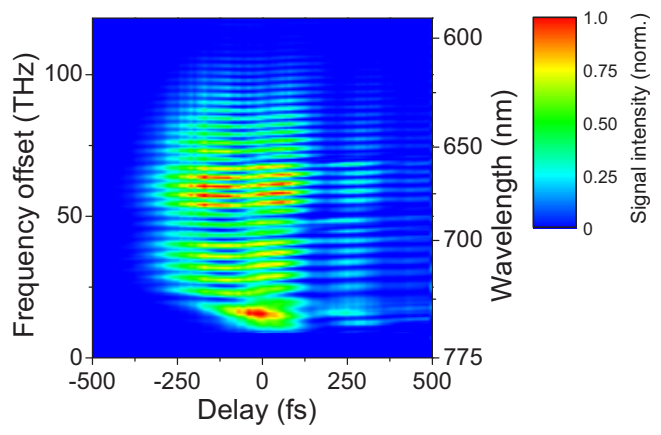

(b)

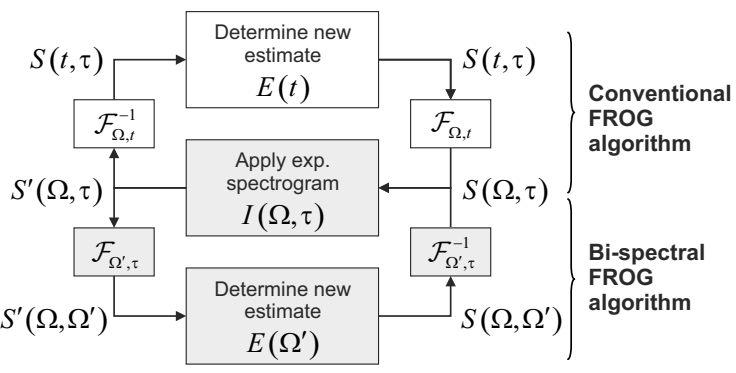

Fig. 1. (a) Experimental spectrogram of an ultra-broadband THz pulse from a two-color air plasma emitter, using a $100-\mu \mathrm{m}$-thick $<110>-\mathrm{GaP}$ crystal and 150 -fs optical pulses for detection. The rapid spectral modulations and temporal broadening are primarily due to variations of the phase-matching response. (b) Schematic of deconvolution algorithm based on bi-spectral domain (conventional time-domain algorithm also included).

close to the phonon resonance of the detection crystal). Moreover, the dithering technique cannot be applied over the full bandwidth (or, not at all, in the case of non-birefringent EO crystals such as ZnTe or $\mathrm{GaP})$.

Fig. 1(a) shows an experimental spectrogram for an ultra-broadband pulse from a two-color plasmaTHz source [1], using a 100- $\mu$ m-thick $<110>$-cut GaP crystal and 150-fs-duration optical pulses for detection. While the underlying THz pulse is only $\sim 100 \mathrm{fs}$ long, the effects of phase-matching lead to a complex spectral and temporal distortion.

It is straightforward to show that the complex spectrogram signal $S(\Omega, \tau)$ is given by:

$$
S(\Omega, \tau)=e^{-i \Omega \tau} \mathcal{F}_{\Omega^{\prime}, \tau}^{-1}\left\{G\left(\Omega, \Omega^{\prime}\right) P\left(\Omega, \Omega^{\prime}\right) E_{\mathrm{opt}}\left(\Omega-\Omega^{\prime}\right) E\left(\Omega^{\prime}\right)\right\}
$$

where $E_{\text {opt }}(\Omega)$ and $E\left(\Omega^{\prime}\right)$ are the complex optical and THz spectra, respectively, and $\mathcal{F}_{\Omega^{\prime}, \tau}^{-1}\{\cdot\} \equiv$ $\int \mathrm{d} \Omega^{\prime} e^{i \Omega^{\prime} \tau}\{\cdot\}$ denotes an inverse Fourier transformation. In Eq. 1 the two response terms,

$$
P\left(\Omega, \Omega^{\prime}\right)=\frac{e^{i \Delta k\left(\Omega, \Omega^{\prime}\right) L}-1}{i \Delta k\left(\Omega, \Omega^{\prime}\right)}, \quad \text { and } \quad G\left(\Omega, \Omega^{\prime}\right)=-i \frac{1}{4 c} \frac{\omega_{0}+\Omega}{n\left(\omega_{0}+\Omega\right)} \chi^{(2)}\left(\Omega^{\prime}, \omega_{0}+\Omega-\Omega^{\prime}\right),
$$

represent the effects of phase-matching and non-linearity, respectively, $\left(\Delta k\left(\Omega, \Omega^{\prime}\right)=k\left(\omega_{0}+\Omega\right)-\right.$ $k\left(\omega_{0}+\Omega-\Omega^{\prime}\right)-k\left(\Omega^{\prime}\right)$ being the wave-vector mismatch and $\omega_{0}$ the optical center frequency). Experimentally, the signal intensity $I(\Omega, \tau)=|S(\Omega, \tau)|^{2}$ is measured. It is the terms $P\left(\Omega, \Omega^{\prime}\right)$ and $G\left(\Omega, \Omega^{\prime}\right)$ which preclude the use of a standard FROG algorithm, via the time-domain signal $S(t, \tau)$, for the recovery.

\section{Generalized deconvolution algorithm}

In order to apply an iterative retrieval algorithm to recover the complex $\mathrm{THz}$ pulse $E(t)$ (or, equivalently, $E\left(\Omega^{\prime}\right)$ ), we note that if we instead Fourier transform $S(\Omega, \tau)$ with respect to $\tau$ (after dropping the $e^{-i \Omega \tau}$ pre-factor, which is not essential as only $|S(\Omega, \tau)|^{2}$ is measured), we obtain a bi-spectral function $S\left(\Omega, \Omega^{\prime}\right)$ equal to the product in the brackets in Eq. 1. Both terms $P$ and $G$ can be calculated from literature data. If the complex optical spectrum $E_{\text {opt }}(\Omega)$ is also available (e.g. from an auxiliary measurement using standard FROG techniques), then one obtains a set of $N_{\tau} \times N_{\Omega^{\prime}}$ equations for the $N_{\Omega^{\prime}}$ unknown values of $E\left(\Omega^{\prime}\right)$. Hence an estimate of $E\left(\Omega^{\prime}\right)$ can be calculated from $S\left(\Omega, \Omega^{\prime}\right)$ using a standard least-squares regression. The complete algorithm proceeds analogously to a standard FROG algorithm, by alternately applying each projection to $S(\Omega, \tau)$, i.e. replacing $|S(\Omega, \tau)|$ with that from 

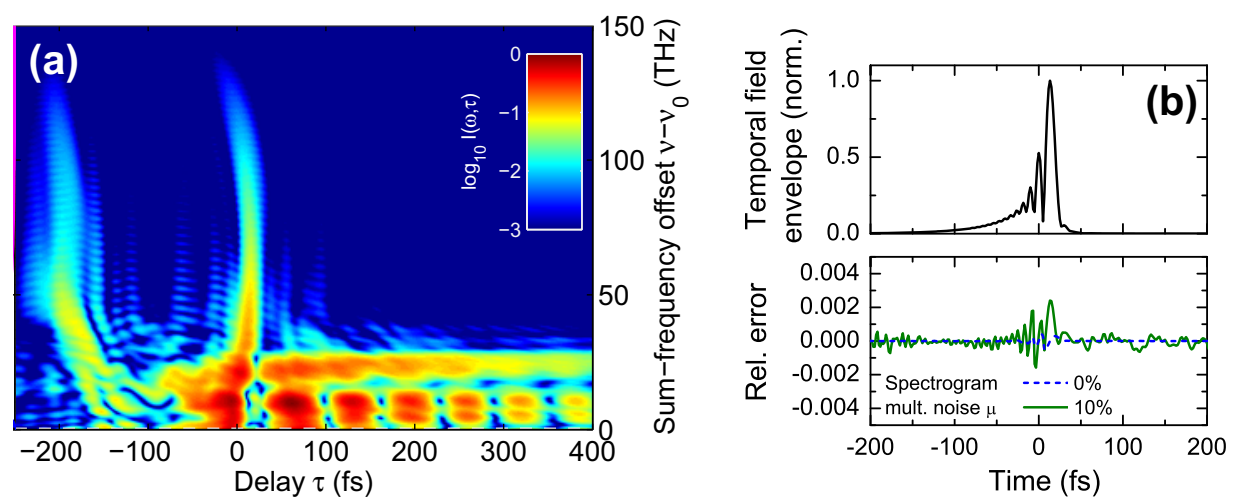

Fig. 2. (a) Numerical test spectrogram for an ultra-broadband THz pulse and $\sim 20$-fs optical gate pulse with a 100 $\mu \mathrm{m}$-thick GaP detection crystal (only sum-frequency range shown). (b) Temporal field envelope and relative error (vs. maximum field) for the recovered field from the noise-free spectrogram $(\mu=0 \%)$ and with $10 \%$ multiplicative noise.

the experimental spectrogram (i.e. $\sqrt{I(\Omega, \tau)})$, and then determining the next estimate of $E\left(\Omega^{\prime}\right)$ and in turn $S\left(\Omega, \Omega^{\prime}\right)$ from Eq. 1. A schematic of the algorithm is shown in Fig. 1(b). While this simple implementation works in principle, in numerical tests of the algorithm we found that it suffers heavily from stagnation at local minima, especially for complex $\mathrm{THz}$ pulses and response functions. Fortunately, this issue could be solved effectively by generalizing the iteration cycle using a hybrid input-output approach [5], which allows the algorithm to escape local minima and converge on the true THz pulse (as confirmed by a wide range of synthetic spectrogram data).

A numerical example of the deconvolution algorithm with a complex spectrogram is shown in Fig. 2, both with and without additional random multiplicative noise $(\mu)$ added to the spectrogram. As shown in Fig. 2(b), the relative error in the retrieved temporal field envelope is well below 1\%, even in the presence of significant multiplicative noise, indicating that the redundancy in the spectrogram promotes a robust recovery (as per the conventional FROG algorithm).

We are currently applying this algorithm to experimental spectrograms measured with such ultrabroadband $\mathrm{THz}$ pulses. Our initial tests are promising, but indicate that a highly accurate determination of the optical gate pulse and crystal dispersion data is necessary in order to recover the $\mathrm{THz}$ field from such complex spectrograms. We note that while we focus here on the measurement of ultra-short THz-mid-infrared pulses, this method may well find application in other wavelength regimes (e.g. $\mathrm{XUV}, \mathrm{X}$-ray) for detection schemes where a complex bi-spectral response function is unavoidable.

\section{References}

1. M.D. Thomson, V. Blank, H.G. Roskos, Opt. Express 18, 23173 (2010)

2. A. Sell, R. Scheu, A. Leitenstorfer, R. Huber, Appl. Phys. Lett. 93, 251107 (2008)

3. S. Akturk, C. D’Amico, A. Mysyrowicz, J. Opt. Soc. Am. B 25, A63 (2008)

4. X. Gu, L. Xu, M. Kimmel, E. Zeek, P. O’Shea, A.P. Shreenath, R. Trebino, Opt. Lett. 27, 1174 (2002)

5. S. Marchesini, Rev. Sci. Instrum. 78, 011301 (2007) 